\title{
Radar Shadow and Superresolution Features for Automatic Recognition of MSTAR targets
}

\author{
Jingjing Cui, Jon Gudnason, Mike Brookes. \\ Imperial College London
}

Key Words: Hidden Markov Model, Target Recognition, High Range Resolution, Synthetic Aperture Radar, Multiple Signal Classification, Feature Extraction, Moving and Stationary Target Acquisition and Recognition (MSTAR)

\section{ABSTRACT}

Automatic target recognition from high range resolution radar profiles remains an important and challenging problem. In this paper, we present a novel feature set for this task that combines a noise-robust superresolution characterisation of the target scattering centres derived using the MUSIC algorithm with a representation of the target's radar shadow shape. To obtain the shadow shape features, three alternative spectral estimation methods are investigated. Using a Hidden Markov Model to represent aspect dependence, we demonstrate that the inclusion of the shadow features results in a significant improvement in recognition performance. Using azimuth apertures of $3^{\circ}$ and $6^{\circ}$ in a 10-target classification task from the MSTAR database, we obtain overall classification error rates of $1.3 \%$ and $0.2 \%$ respectively. These results are significantly better than those obtained by other published methods on the same database.

\section{INTRODUCTION}

The automatic detection and classification of targets from their radar signatures is an important and difficult problem that has attracted considerable research effort. Algorithms for target recognition from high range resolution (HRR) radar signals generally use as their primary input either a synthetic aperture radar (SAR) image or else a sequence of one or more one-dimensional HRR range profiles. The image-based approaches generally have higher performance but are less robust to target motion because of their long data acquisition time. Some image-based algorithms use the pixel values of the image as their recognition features $[1,2,3]$ while others first transform the image to another domain $[4,5]$. An alternative approach for targets that are large compared with the radar wavelength is to model the radar return as emanating from a discrete set of orientation-dependent points known as scattering centres [6]. In this approach, the SAR image is processed to generate an explicit list of scattering centre positions and associated radar cross sections on which the recognition features are based [7, 8]. In the same way, systems that act on the HRR range profiles can either use the raw [9] or transformed [10] profile values as their features or else can process the profiles to estimate the scattering centre locations and cross sections [11]. Both SAR images and HRR profiles can exhibit large variations for small changes in target orientation. Target recognition systems must account for this aspect-dependency by using a rotation invariant transform [4] or by having multiple, orientation-dependent, target representations which may conveniently be embedded in a Hidden Markov Model (HMM) [5, 11, 12].

In this paper, we present a novel feature set for automatic target recognition from a sequence of radar range profiles. Our feature set uses a noise-robust super-resolution technique for identifying scattering centre locations and combines this information with additional features that characterise the shape of the radar shadow. Fig. 1(b) shows a SAR image of a T72 tank taken from the MSTAR $[13,14]$ dataset. This image may be divided into three regions having significantly different characteristics: (a) the target itself, (b) the target shadow and (c) a clutter region surrounding the target. As can be seen in this example, the shape of the shadow region gives potentially useful information about the vertical profile of a target when it is sited on level ground. This information is not available from the direct target returns which are insensitive to vertical displacement. The shadow information has been used by others to improve target detection [15] but is not generally used explicitly in target recognition. We showed in [16] that the shadow information can be effective in radar target recognition and we present further improvements in performance in the present paper.
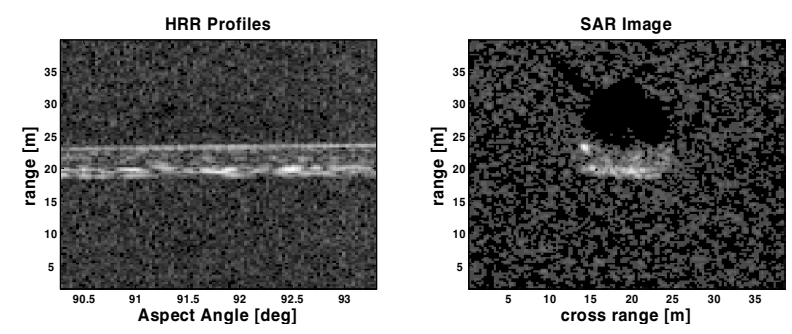

Fig.1. (a) HRR profiles (b) SAR image of T72 tank

In Section 2 of this paper, we describe our proposed feature set in detail and in Section 3, we describe the Hidden Markov model that we use to represent the aspect dependency of the radar returns. In Section 4, we evaluate the performance of our target recognition system using observation data from the MSTAR database [14] and compare its performance with that of other systems from the literature that use the same database. Finally, we summarise our results in Section 5. 


\section{RECOGNITION FEATURE SET}

The features that we use for target recognition are derived from the sequence of complex-valued HRR profiles, $x(n, k)$, obtained by applying a discrete Fourier transform (DFT) to the windowed phase history radar returns. Here $n$ is the profile index and $k$ is the range-bin index covering the region of interest. Fig. 1(a) shows a typical plot of $x(n, k)$ and Fig. 1(b) shows the SAR image that results from windowing $x(n, k)$ and taking the DFT with respect to $n$. Visible in the image are the target itself, with signal levels well above the clutter noise level and also a well defined shadow region with very low signal levels. For each value of the profile index $n$, we obtain two feature vectors: $\mathbf{u}(n)$ characterizes the positions and intensities of the scattering centres within the target while $\mathbf{w}(n)$ characterises the shape of the shadow area. Both these feature vectors are derived from $2 P+1$ consecutive profiles centred on profile $n$. We therefore define the data matrix $x_{n}(p, k)=x(n+p, k), \quad$ where $p \in\{-P, \ldots, P\} . \quad$ The procedures to obtain $\mathbf{u}(n)$ and $\mathbf{w}(n)$ are described below.

\subsection{Target features}

The derivation of $\mathbf{u}(n)$ is illustrated in Fig. 2 and the processing steps are described below where, for clarity, we omit the profile index, $n$.

\begin{tabular}{|c|c|c|c|c|c|c|c|c|}
\hline$x(p, k)$ & range & & $a_{m, k}$ & & $y(l, k)$ & $\log$ & select low & $\mathbf{u}$ \\
\hline & target & range & $w_{m, k}$ & mask & & dct & components & \\
\hline
\end{tabular}

Fig.2. Procedure to calculate target features from HRR profiles

Each scattering centre in range bin $k$ gives rise to a complex exponential term in $x(n, k)$ and the first step in obtaining $\mathbf{u}$ is to identify these terms. We do this by applying the MUSIC algorithm [17] which uses the data model:

$$
x(p, k)=\sum_{m=1}^{M} \alpha_{m, k} e^{j \omega_{m, k} p}+\varepsilon(p, k)
$$

where $\alpha_{m, k}$ and $\omega_{m, k}$ are the complex amplitudes and frequencies of the scattering centre terms and $\varepsilon(p, k)$ is assumed to be white noise. The reasons for using the MUSIC algorithm are that it is resistant to noise, does not require windowing of $x(n, k)$ and is able to estimate $\omega_{m, k}$ with high resolution independently of $P$. Within range bin $k$, each of the $M$ components in (1) corresponds to a scattering centre whose cross-range displacement is proportional to $\omega_{m, k}$. The maximum number of scattering centres, $M$, could be chosen adaptively for each range bin but we have, in the experiments below, fixed it for each range bin according to the number of HRR profiles used.

After discarding any scattering centres whose cross-range displacement lies outside the target mask, we convert the continuous displacements, $\omega_{m, k}$, to discrete values. We create a continuous signal containing an impulse for each scattering centre which we then low-pass filter and sample to give:

$$
y(l, k)=\sum_{m=1}^{M}\left|\alpha_{m, k}\right|^{2} h\left(l-\beta \omega_{m, k}\right)
$$

where $h(l)=(0.33 \pi l)^{-1} \sin (0.33 \pi l)$ is the low-pass filter response and $\beta=0.5 \lambda(2 \pi \Delta \phi \Delta r)^{-1}$ is a constant with $\lambda, \Delta \phi$ and $\Delta r$, the wavelength, azimuth increment and cross range resolution, respectively. Finally, the image information is compressed by taking the 2-dimensional discrete cosine transform (DCT) of $\log y(l, k)$ and we form a 54-element feature vector $\mathbf{u}$, retaining only the coefficients in the low frequency triangle of size 10-by-10 and excluding the DC term. Fig. 3(a) shows an example of $y(l, k)$ corresponding to the central portion of the image shown in Fig. 1(b) and Fig. 3(b) shows the reconstructed logarithmic image using only the coefficients for $\mathbf{u}$.
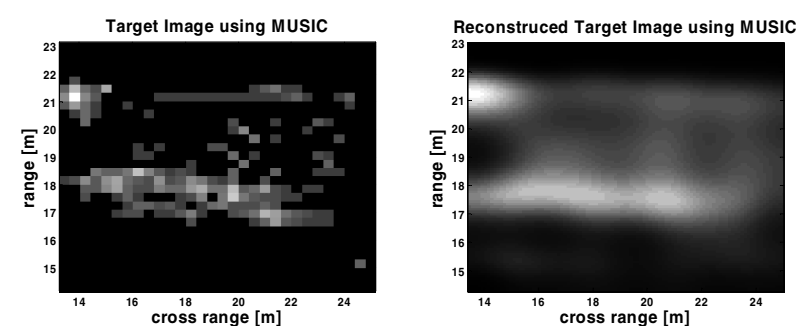

Fig. 3 (a) The image $y(l, k)$ of the T72 tank (b) The reconstructed logarithmic image using $\mathbf{u}$

\subsection{Shadow features}

The shadow area of the SAR image consists of deep valleys corresponding to the shadow and broad peaks representing the clutter. Unlike the target area of the SAR image, the shadow region contains no sharp peaks from scattering centres and we wish to characterise the shape of its boundary. The procedure for obtaining the shadow feature vector, $\mathbf{w}$, is shown in Fig. 7. Following a range mask, we perform spectral estimation in the cross-range direction using one of three methods described below. We then identify the shadow region using an adaptive threshold and, as with the target features, compress the information using a DCT.

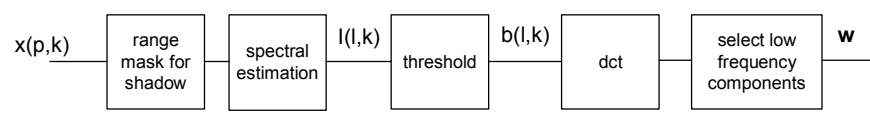

Fig.7 Procedure to calculate shadow features from HRR profiles using one of the three spectral estimation methods

\subsubsection{Fourier Transform spectral estimation}

The first method of spectral estimation is to apply a Hamming window to $x(p, k)$ and then to take the DFT in the $p$ direction. The shape of the spectrum is thus estimated for each range bin $k$ and the image $I(l, k)$ is formed in the same 
way as a SAR image. A drawback of this method is that the cross-range resolution is limited by the windowing operation.

\subsubsection{Autoregressive spectral estimation}

A better spectral estimate is achieved by deriving the parameters of a model of the cross range profile. First we apply the autocorrelation method of autoregressive (AR) spectral estimation to $x(p, k)$ using the model:

$$
x(p, k)=-\sum_{m=1}^{M} a_{m}(k) x(p-m, k)+u(p, k)
$$

where $a_{m}(k)$ are complex AR coefficients and $u(p, k)$ is a white input driving sequence of zero mean and unit variance. We then form the image $I(l, k)$ by converting the AR coefficients $a_{m}(k)_{m=1}^{M}$ to the spectrum in the $p$ direction:

$$
I(l, k)=\frac{1}{\sum_{m=1}^{M} a_{m}(k) e^{-j 2 \pi l m}}
$$

The AR model is an all-pole model and the model order, $M$, is fixed at 25 in the experiments below. The model order is high because the peaks in the resulting spectrum will model the clutter around the shadow even though the recognition is not done on these peaks but on the shape of the shadow.

\subsubsection{Moving average spectral estimation}

We can also estimate the spectrum of $x(p, k)$ by applying a Moving Average (MA) model:

$$
x(p, k)=\sum_{m=0}^{M} b_{m}(k) u(p-m, k)
$$

where $b_{m}(k)$ are complex MA coefficients and $u(p, k)$ is the input driving sequence. The MA model is an all-zero model which characterises the shadow. The $\operatorname{model} \operatorname{order} M$, in the experiments below, fixed at 2 , in order to locate edge of shadow. We form the image $I(l, k)$ by converting the MA coefficients $b_{m}(k)_{m=0}^{M}$ to the spectrum in the $p$ direction:

$$
I(l, k)=\sum_{m=0}^{M} b_{m}(k) e^{-j 2 \pi l m}
$$

To estimate the MA coefficients, we first convert the process to a high-order AR process [18] and then use standard AR estimation procedures to obtain the MA coefficients. The method uses the following model:

$$
\begin{gathered}
x(p, k)=u(p, k)-\sum_{n=1}^{N} a_{n}(k) x(p-n, k) \quad(N>>M) \\
v(p, k)=\left\{\begin{array}{cc}
1 & \text { for } p=1 \\
a_{p-1}(k) & \text { for } 1<p \leq N+1 \\
0 & \text { for } p>N+1
\end{array}\right. \\
v(p, k)=u^{\prime}(p, k)-\sum_{m=1}^{M} b_{m}(k) v(p-m, k)
\end{gathered}
$$

In our implementation we used $N=4 \times M$.

\subsubsection{Binary Image Formation}

After obtaining the image $I(l, k)$ from the shadow mask, we threshold it to give a binary-valued shadow image $b(l, k)$ shown in Fig. 5 for each of the spectral estimation methods. The pixel values representing the clutter region in $I(l, k)$ have a large spread whereas the shadow pixels are concentrated in lower values. This allows us to determine the threshold from the histogram by choosing it to be above the highest value of the bins containing the most pixels.

The shadow images in Fig. 5 are obtained from the data shown in Fig. 1 using (a) the Fourier transform, (b) AR spectral estimation and (c) MA spectral estimation. The figures show that the shadow is featured prominently as a large black area while the clutter is shown as mixed points of black and white. As with the target features, we compress the shadow image by taking a 2-dimensional DCT and retaining 54 low frequency coefficients to form the shadow feature vectors denoted by $\mathbf{w}_{F}, \mathbf{w}_{A}$ and $\mathbf{w}_{M}$ respectively for the three methods. The reconstructed images using $\mathbf{w}_{F}, \mathbf{w}_{A}$ and $\mathbf{w}_{M}$ are shown in Fig. 6. The shapes of the shadows are all well retained except that the MA method introduces some horizontal steaks.
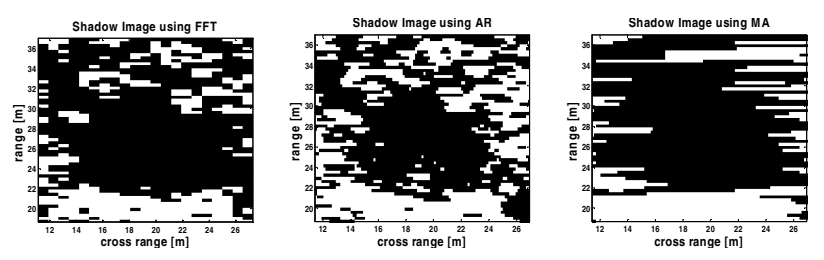

Fig. 5 The image $b(l, k)$ using (a) Fourier transform, (b) AR spectral estimation, (c) MA spectral estimation.
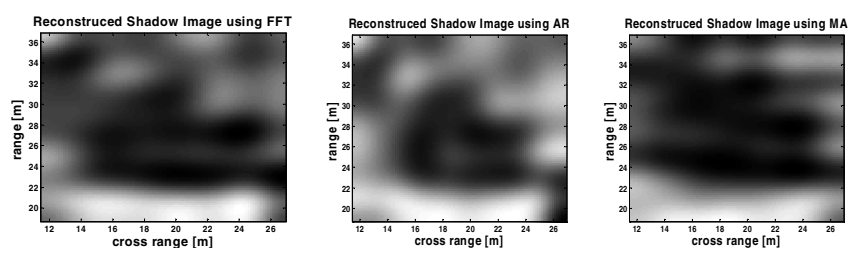

Fig. 6 Reconstructed images from (a) $\mathbf{w}_{F}$, (b) $\mathbf{w}_{A}$, (c) $\mathbf{w}_{M}$

The number, $2 P+1$, of HRR profiles used to form the feature vector has a direct effect on the cross-range resolution of $\mathbf{w}_{F}$ and $\mathbf{w}_{A}$ but not on that of $\mathbf{w}_{M}$. However although AR spectral estimation can achieve superresolution, its error criterion concentrates on the high energy features in the clutter region and so does not find the shadow edges accurately. In contrast the MA approach represents the shadow region well even using only a $2^{\text {nd }}$ order model. We compare the classification performances using the three kinds of features in the Section 4. 


\section{AZIMUTH HIDDEN MARKOV MODEL}

HRR profiles exhibit significant variability with target orientation. We model this for each target using an HMM containing $S$ states which correspond to different target aspects. Within an observation sequence, consecutive HRR profiles correspond either to the same or to adjacent states. Thus the only allowable state transitions are from a state to itself or to the adjacent state in the direction of sensor motion.

We initialise the states to correspond to equal aspect increments of $360^{\circ} \mathrm{S}^{-1}$ and for each state we train a Gaussian mixture model (GMM) [19] using all available training data from the corresponding range of aspects of a particular target. The transition probability between adjacent states is initialised to be $S \Delta \phi / 360^{\circ}$ where $\Delta \phi$ is the azimuth increment between successive feature vectors. Using these initial values, we then re-estimate the GMM parameters and the HMM transition probabilities using embedded Baum-Welch training [20].

The azimuth interval represented by a state can change during re-estimation as is illustrated in Fig. 8(a). This figure shows the log likelihood of test feature vectors as a function of azimuth angle for each of three consecutive model states. The three model states were initially trained with data from consecutive $6^{\circ}$ azimuth intervals in the region of $60^{\circ}$. We see from Fig. 8(a) that the log likelihood does indeed peak at a target orientation of $60^{\circ}$ and that there is a second, smaller,

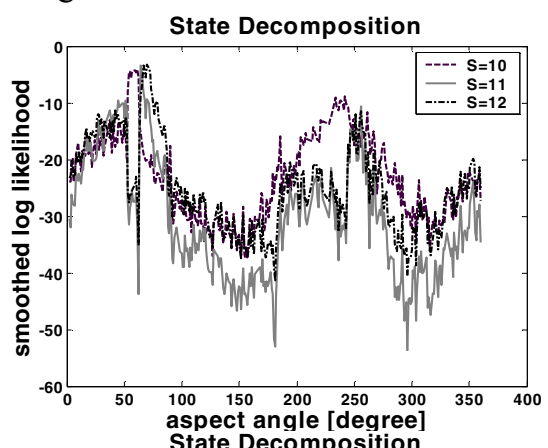
peak at around $250^{\circ}$ due to target symmetry. An enlarged view of the primary peak is shown in Fig. 8(b) which shows that each state has retained clearly defined boundaries.

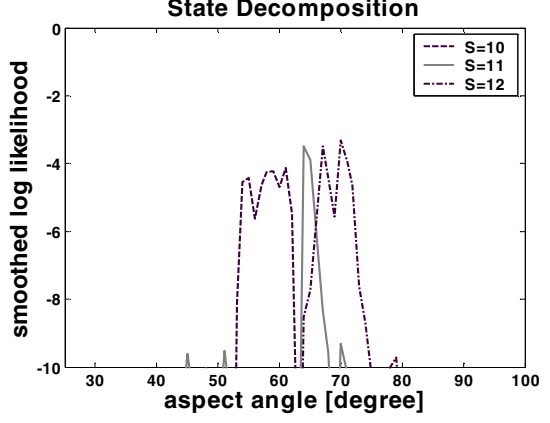

State 10 is however much broader than its initial width of $6^{\circ}$ and now covers about $10^{\circ}$, state 11 covers a relatively narrower angular extent about $2^{\circ}$ and state 12 becomes broader again.

Fig. 8 State decomposition (a) from $0^{\circ}$ to $360^{\circ}$ (b) from $25^{\circ}$ to $100^{\circ}$

\section{EXPERIMENTAL RESULTS}

\subsection{MSTAR database}

The experimental evaluations make use of the Moving and Stationary Target Acquisition and Recognition (MSTAR) database collected by the Sandia National Laboratory using an $\mathrm{X}$-band SAR sensor in $0.3 \mathrm{~m}$ resolution spotlight mode [14, 13]. The database contains complex valued SAR image chips of 10 confusable targets and their variants. For each target, the images cover a full $360^{\circ}$ azimuth range at depression angles of $15^{\circ}$ and $17^{\circ}$ for test and training data respectively. The SAR images have a resolution of $\Delta r=0.3 \mathrm{~m}$ in both the range (horizontal) and cross range (vertical) directions.

For our experiments, the SAR image chips were converted into a sequence of HRR profiles by taking an inverse Fourier transform, removing the zero padding and finally undoing the Taylor window in the $P$ direction. The steps of this procedure are summarised in Fig. 9. Each image chip covers an azimuth interval of approximately $3^{\circ}$ [9] with successive HRR profiles separated by an angular increment of $\Delta \phi=0.03^{\circ}$.

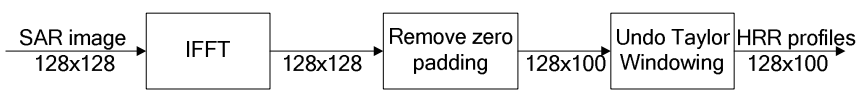

Fig.9. Procedure to convert MSTAR images to HRR profiles. The operations are in cross-range.

\subsection{Experimental procedure}

For our experiments we used an HMM containing $S=60$ states each corresponding to an initial azimuth interval of $6^{\circ}$. Within each state, feature vector distributions are represented by a diagonal-covariance Gaussian. We used a $9 \mathrm{~m} \times 9 \mathrm{~m}$ target mask and a $18 \mathrm{~m} \times 9 \mathrm{~m}$ shadow mask. A total of 3000 image chips containing $17^{\circ}$ depression angle data was used to train a separate HMM for each of the targets. For testing, we used a total of $500015^{\circ}$ depression angle images without any compensation for the slight mismatch in depression angle.

We evaluated three alternative feature sets: the target features $\mathbf{u}$, the shadow features $\mathbf{w}$ and the concatenation of the two $\mathbf{u}+\mathbf{w}$.The first two sets contain 54 elements while the last has 108. The feature vectors were formed using $P=25$ corresponding to an aperture of $1.5^{\circ}$.The maximum number of scattering centres, $M$, is fixed at 10 for each range bin. We formed a sequence of HRR profiles covering $3^{\circ}$ azimuth aperture from one image chip and a sequence covering $6^{\circ}$ from two adjacent image chips. We then determined the model with the highest likelihood. Model training and recognition were performed using the HTK recognition software [20].

We are interested in knowing what method to use for extracting the shadow features. On their own, the shadow features do not perform well, but in conjunction with the target features, they can improve the recognition results significantly. Table 1 shows a closed-set identification results using the target features $\mathbf{u}$, and three proposed shadow features $\mathbf{w}_{F}, \mathbf{w}_{A}$ and $\mathbf{w}_{M}$ used both on their own and concatenated with $\mathbf{u}$ to form a 108 element feature vector. The table presents the percentage misclassification rates for each 
individual target, the overall test set misclassification rate (MCR) and the standard deviation of the test set misclassification rate (St.Dev) using test sequences which each cover a $3^{\circ}$ azimuth aperture. We can see that if used on their own, the AR derived shadow features perform best with $7.3 \%$ MCR. However, when used with the target features, the MA derived shadow features produce the best performance of $1.3 \% \mathrm{MCR}$. The reason for this is that the shadow mask overlaps the target region and both $\mathbf{w}_{F}$ and $\mathbf{w}_{A}$ manage to include information that is already in $\mathbf{u}$. The MA features on the other hand concentrate on the shadow, giving relatively poor performance on their own but giving the greatest improvement when combined with the target features, $\mathbf{u}$. We will use $\mathbf{w}_{M}$ to represent the shadow features in the following discussions.

Table 1: Recognition error rates with different shadow features (\%)

\begin{tabular}{|c|c|c|c|c|c|c|c|}
\hline Target & $\mathbf{u}$ & $\mathbf{w}_{\mathrm{F}}$ & $\mathbf{u}+\mathbf{w}_{\mathrm{F}}$ & $\mathbf{w}_{\mathrm{A}}$ & $\mathbf{u}+\mathbf{w}_{\mathrm{A}}$ & $\mathbf{w}_{\mathrm{M}}$ & $\mathbf{u}+\mathbf{w}_{\mathbf{M}}$ \\
\hline \hline BMP2 & 3.6 & 34.6 & 3.1 & 19.1 & 0.5 & 42.8 & 1 \\
\hline BRDM2 & 12.8 & 14.9 & 6.2 & 11.7 & 4.7 & 17.5 & 3.7 \\
\hline BTR60 & 0 & 14.5 & 0 & 6.7 & 0 & 33.2 & 1.6 \\
\hline BTR70 & 4.1 & 26.8 & 8.3 & 17 & 4.6 & 27.8 & 2.1 \\
\hline D7 & 1.8 & 1.8 & 1.1 & 1.1 & 0.7 & 3.7 & 0.4 \\
\hline T62 & 6.6 & 1.8 & 1.1 & 2.9 & 1.5 & 8.1 & 0.4 \\
\hline T72 & 1.6 & 18 & 1.6 & 17.5 & 0.5 & 30.4 & 0 \\
\hline ZIL131 & 1.8 & 0 & 0 & 0.4 & 0 & 0 & 0 \\
\hline ZSU234 & 1.5 & 0.4 & 0.4 & 0.7 & 0.4 & 8.4 & 0.7 \\
\hline 2S1 & 5.8 & 4 & 2.9 & 5.1 & 2.9 & 12.4 & 2.9 \\
\hline \hline MCR & 4.2 & 10.1 & 2.4 & 7.3 & 1.6 & 16.4 & 1.3 \\
\hline St.Dev. & 0.4 & 0.6 & 0.3 & 0.5 & 0.3 & 0.8 & 0.2 \\
\hline
\end{tabular}

We now present the recognition results based on $3^{\circ}$ and $6^{\circ}$ sequences, respectively. Table 2 shows the recognition error rate for each of the three feature sets for the two cases.

In the $6^{\circ}$-sequence observation based recognition, the log likelihood of two adjacent test sequences are added and the recogniser chooses the one with the highest sum. We see that used on their own the $\mathbf{u}$ and $\mathbf{w}$ parameter sets give MCR of $1.5 \%$ and $10.9 \%$ which is in both cases an improvement over the one-sequence results. When the two feature sets are combined to form $\mathbf{u}+\mathbf{w}$ we find that the MCR is reduced to $0.2 \%$ with seven of the ten targets error-free.

Table 2: Recognition error rates of two observations (\%)

\begin{tabular}{|c|c|c|c|c|c|c|}
\hline & \multicolumn{3}{|c|}{$3^{\circ}$ azimuth aperture } & \multicolumn{3}{|c|}{$6^{\circ}$ azimuth aperture } \\
\hline Target & $\mathbf{u}$ & $\mathbf{w}$ & $\mathbf{u}+\mathbf{w}$ & $\mathbf{u}$ & $\mathbf{w}$ & $\mathbf{u + w}$ \\
\hline \hline BMP2 & 3.6 & 42.8 & 1 & 2 & 30 & 0.5 \\
\hline BRDM2 & 12.8 & 17.5 & 3.7 & 3.3 & 9.2 & 0 \\
\hline BTR60 & 0 & 33.2 & 1.6 & 0 & 26 & 0.5 \\
\hline BTR70 & 4.1 & 27.8 & 2.1 & 1 & 16.1 & 1 \\
\hline D7 & 1.8 & 3.7 & 0.4 & 1.1 & 0.7 & 0 \\
\hline T62 & 6.6 & 8.1 & 0.4 & 2.2 & 2.9 & 0 \\
\hline T72 & 1.6 & 30.4 & 0 & 1 & 25.4 & 0 \\
\hline ZIL131 & 1.8 & 0 & 0 & 0 & 0 & 0 \\
\hline ZSU234 & 1.5 & 8.4 & 0.7 & 1.1 & 4.8 & 0 \\
\hline 2S1 & 5.8 & 12.4 & 2.9 & 2.6 & 9.5 & 0 \\
\hline \hline MCR & 4.2 & 16.4 & 1.3 & 1.5 & 10.9 & 0.2 \\
\hline St.Dev. & 0.4 & 0.8 & 0.2 & 0.3 & 0.6 & 0.08 \\
\hline
\end{tabular}

For both the $3^{\circ}$-sequence and $6^{\circ}$-sequence tests, the addition of the $\mathbf{w}$ features improves the recognition rate for all targets except one, the BTR60 which is considerably worsened. This indicates that, for this target at least, the assumption that the feature vector follows a multivariate Gaussian distribution is a poor one.

The error rate of $1.3 \%$ obtained using a $3^{\circ}$ aperture can be directly compared with other published results based on the MSTAR database with the same recognition task. In [21], the authors obtained error rates of $4.1 \%$ using an approach based on the SAR image and in [11] an error rate of $17.8 \%$ was obtained when performing recognition on the HRR profiles directly.

The MSTAR database contains 11 variants of the T72 tank and 3 variants of the BMP2 vehicle, manifested by different realisations of the fuel tank, antenna, etc [11]. To evaluate the robustness of our recogniser to these variations, we trained models on two BMP2 variants, four T72 variants as well as the eight other targets using the $\mathbf{u}+\mathbf{w}$ feature set. We then conducted recognition tests on all 22 targets in the database using two images. If the recogniser identified an incorrect variant of the correct tank model, it was counted as an error in the "Strict" column of Table 3 but as a correct identification in the "Class" column.

For targets included in the training set, the MCR is $0.4 \%$ and in most cases the precise variant of a particular target is identified correctly. For the unseen variants listed in the lower section of the table, the MCR was $8.6 \%$ with more than half of the errors arising from the T72-s7 and T72-812 targets.

The final column of Table 3 shows the "Class" error rates reported in [11] for the same task. We see that for all targets except T72-s7 the recognition performance for our proposed feature set is considerably better. We note however that since [11] bases its recognition on individual HRR profiles, it will be less sensitive to target motion than when the feature set described here is used with a large value of $P$.

Table 3: Recognition error rates with unknown targets (\%)

\begin{tabular}{|c|c|c|c|}
\hline Target & Strict & Class & $\begin{array}{c}\text { Class from } \\
{[11]}\end{array}$ \\
\hline \hline BMP2-c21 & 13 & 4.7 & 6.1 \\
\hline BMP2-9563 & 7.3 & 0 & 7 \\
\hline BTR70 & 1 & - & 14.1 \\
\hline BRDM2 & 0 & - & 8.6 \\
\hline BTR60 & 0.5 & - & 13.7 \\
\hline D7 & 0 & - & 4.8 \\
\hline T62 & 0 & - & 9 \\
\hline T72-a04 & 1.5 & 0 & 2.9 \\
\hline T72-a10 & 0.4 & 0 & 3.4 \\
\hline T72-a62 & 0.4 & 0 & 3.9 \\
\hline T72-132 & 4.1 & 0 & 7.6 \\
\hline ZIL131 & 0 & - & 10.3 \\
\hline ZSU234 & 0 & - & 8.5 \\
\hline 2S1 & 0 & - & 4.7 \\
\hline \hline BMP2-9566 & - & 0.5 & 37.5 \\
\hline T72-a05 & - & 7 & 14.5 \\
\hline T72-a07 & - & 2.6 & 16.1 \\
\hline T72-a32 & - & 1.5 & 22.9 \\
\hline T72-a63 & - & 1.8 & 15.9 \\
\hline T72-a64 & - & 5.1 & 31.7 \\
\hline T72-s7 & - & 27.3 & 17.1 \\
\hline T72-812 & - & 23.4 & 29.6 \\
\hline
\end{tabular}

\section{CONCLUSIONS}

This paper has presented a novel radar target recognition technique combining two-dimensional target and shadow information. The new technique complements the SAR-ATR and HRR-ATR techniques by using a feature extraction 
method that is robust to noise and that can extract target and shadow information accurately with limited azimuth aperture length. Three methods to obtain the shadow features are investigated. The experimental results using MSTAR database indicate that although they perform relatively poorly on their own, the shadow features derived using the MA method perform exceptionally well when combined with MUSICderived target features. Using azimuth apertures of $3^{\circ}$ and $6^{\circ}$ in a 10-target classification task, they give overall classification error rates of $1.3 \%$ and $0.2 \%$ respectively. These results are considerably better than other published techniques using the same dataset.

\section{ACKNOWLEDGEMENT}

This research was supported by the UK MoD through work funded by the Defence Technology Centre for Data and Information Fusion.

\section{REFERENCES}

1. L. M. Novak, "A comparison of $1 \mathrm{D}$ and $2 \mathrm{D}$ algorithms for radar target classification", IEEE International Conference on Systems Engineering, 1991, pp. 6-21.

2. L. M. Novak, S. D. Halversen, G. Owirka, and M. Hiett, "Effects of polarization and resolution on SAR ATR", IEEE Trans. on Aerospace and Electronic Systems, vol. 33, no. 1, 1997, pp. 102-116.

3. J. A. O'Sullivan, M. D. DeVore, V. Kedia, and M. I. Miller, "SAR ATR performance using a conditionally Gaussian model", IEEE Trans. On Aerospace and Electronic Systems, vol. 37, no. 1, 2001, pp. 91-108.

4. D. P. Kottke, F. Jong-Kae, and K. Brown, "Hidden Markov modeling for automatic target recognition", Proc. Thirty-First Asilomar Conference on Signals, Systems and Computers, vol. 1. 1997, pp. 859-863.

5. P. Runkle, L. H. Nguyen, J. H. McClellan, and L. Carin, "Multi-aspect target detection for SAR imagery using hidden Markov models", IEEE Trans. On Geoscience and Remote Sensing, vol. 39, no. 1, 2001, pp. 46-55.

6. R. Bhalla, H. Ling, J. Moore, D. J. Andersh, S. W. Lee, and J. Hughes, "3D scattering centre representation $\mathrm{f}$ complex targets using the shooting and bouncing ray technique: a review", IEEE Antennas and Propagation Magazine, vol. 40, no. 5, 1998, pp. 30-39.

7. H. Chiang, R. L., Moses, and L. C. Potter, "Model-based classification of radar images", IEEE Trans. on Information Theory, vol. 46, no. 5, 2000, pp.1842-1854.

8. C. Nilubol, Q. H. Pham, R. M. Mersereau, M. J. T. Smith, and M. A. Clements, "Hidden Markov modelling for SAR automatic target recognition", Proc. IEEE Int. Conf. Acoustics, Speech, Signal Processing, vol. 2, 1998, pp. 1061-1064.

9. R. Williams, J. Westerkamp, D. Gross, A. Palomion, and T. Fister, "Automatic target recognition of time critical moving targets using $1 \mathrm{D}$ high range resolution (HRR) radar", IEEE Radar Conference, 1999, pp. 54-59.

10. D. E. Nelson, J. A. Starzuk, and D. D. Ensley, "Iterated wavelet transformation and signal discrimination for HRR radar target recognition", IEEE Trans. on Systems, Man and Cybernetics, Part A, vol. 33, no. 1, 2003, pp. 52-57.

11. X. Liao, P. Runkle, and L. Carin, "Identification of ground targets from sequential high-range-resolution radar signatures", IEEE Trans. On Aerospace and Electronic Systems, vol. 38, no. 4, 2002, pp. 1230-1242.

12. B. Pei and Z. Bao, "Radar target recognition based on peak location of HRR profile and HMMs classifiers", Proc. Radar Conference, 2002, pp. 414-418.

13. T. D. Ross and J. C. Mossing, "The MSTAR Evaluation Methodology", Proc. SPIE - The International Society for Optical Engineering, vol. 3721, 1999, pp. 705-713.

14. T. Ross, S. Worrel, V. Velten, J. Mossing, and M. Bryant, "Standard SAR ATR evaluation experiments using the MSTAR public release data set", Proc. SPIE - The International Society for Optical Engineering, vol. 3370, 1998, pp. 556-573.

15. P. Lombardo, M. Sciotti, and L. M. Kaplan, "SAR prescreening using both target and shadow information", Proc. IEEE Radar Conference, 2001, pp. 147-152.

16. J. Cui, J. Godnason and M.Brookes, "Automatic Recognition of MSTAR Targets using Radar Shadow and superresolution Features", is accepted by Proc. IEEE Int. Conf. Acoustics, Speech, Signal Processing, 2005.

17. R. Schmidt, "Multiple emitter location and signal parameter estimation", IEEE Trans. on Antennas and Propagation, vol. 34, no. 3, 1986, pp. 276-280.

18. S. Kay, Modern spectral estimation, $1^{\text {st }}$ ed. Prentice Hall, 1988.

19. A. P. Dempster, N. M. Laird, and D. B. Rubin, "Maximum Likelihood from Incomplete Data via the EM Algorithm", Journal of the Royal Statistical Society, vol. 39, no. 1, 1977, pp. 1-38.

20. S. Young, G. Evermann, T. Hain, D. Kershaw, G. Moore, J. Odell, D. Ollason, D. Povey, V. Valtchev, and P. Woodland, The HTK Book, Cambridge University Engineering Dept, 2002. [Online] Available: http://htk.eng.cam.ac.uk/protdocs/HTKBook/htkbook.html

21.L. M. Novak, "Automatic target recognition using enhanced resolution SAR data", IEEE Trans. on Aerospace and Electronic Systems, vol. 35, no. 1, 1999, pp. 157-175.

\section{BIOGRAPHY}

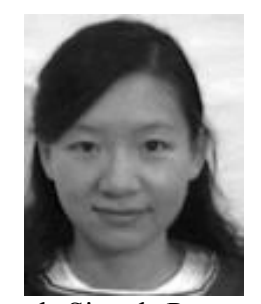

Jingjing Cui was born in 1980. She received her B. S. degree from the Department of Communication Engineering in Fudan University, China, in 2002, and in 2003 she received her MSc degree from the Department of Electrical and Electronic Engineering in Imperial College London, UK. She is currently doing her $\mathrm{PhD}$ degree at the Communication and Signal Processing Group in Imperial College. Her current research interests include echo cancellation and radar target recognition. Her contact address is: Communication and Signal Processing Group, Department of Electrical and Electronic Engineering, Imperial College London, UK, SW7 2BT. 